\title{
Correction: PRMT1 suppresses ATF4-mediated endoplasmic reticulum response in cardiomyocytes
}

Myong-Ho Jeong, Hyeon-Ju Jeong, Byeong-Yun Ahn, Jung-Hoon Pyun, Ilmin Kwon, Hana Cho and Jong-Sun Kang

\section{Correction to: Cell Death \& Disease}

https://doi.org/10.1038/s41419-019-2147-3

published online 02 December 2019

Following publication of this article, the authors found a numerical notation error in Fig. 3B. 636 should have read
240, and 643 should have read 263.This has been corrected in the PDF and HTML versions.

Published online: 23 March 2020 